BNL-73499-2005-CP

\title{
Electromigration Issues in High Current Horn
}

\author{
W. Zhang, S. Bellavia, J. Sandberg, N. Simos, J. Tuozzolo, \\ W-T. Weng, et al
}
Presented at the Particle Accelerator Conference(PAC'05) Knoxville, Tennessee
May 16-20, 2005

\author{
Collider-Accelerator Department \\ Brookhaven National Laboratory \\ P.O. Box 5000 \\ Upton, NY 11973-5000 \\ www.bnl.gov \\ Managed by \\ Brookhaven Science Associates, LIC \\ for the United States Department of Energy under \\ Contract No. DE-AC02-98CH10886
}

This is a preprint of a paper intended for publication in a journal or proceedings. Since changes may be made before publication, this preprint is made available with the understanding that it will not be cited or reproduced without the permission of the author. 


\section{DISCLAIMER}

This report was prepared as an account of work sponsored by an agency of the United States Government. Neither the United States Government nor any agency thereof, nor any of their employees, nor any of their contractors, subcontractors, or their employees, makes any warranty, express or implied, or assumes any legal liability or responsibility for the accuracy, completeness, or any third party's use or the results of such use of any information, apparatus, product, or process disclosed, or represents that its use would not infringe privately owned rights. Reference herein to any specific commercial product, process, or service by trade name, trademark, manufacturer, or otherwise, does not necessarily constitute or imply its endorsement, recommendation, or favoring by the United States Government or any agency thereof or its contractors or subcontractors. The views and opinions of authors expressed herein do not necessarily state or reflect those of the United States Government or any agency thereof.

FOR UNCLASSIFIED, UNLIMITED STI PRODUCTS

Available electronically at:

OSTI:

http://www.osti.gov/bridge

Available for a processing fee to U.S. Department of Energy and its contractors, in paper from:
U.S. Department of Energy
Office of Scientific and Technical Information
P.O. Box 62
Oak Ridge, TN 37831
Phone: (865) 576-8401
Facsimile: (865) 576-5728
E-mail: reports@adonis.osti.gov

National Technical Information Service (NTIS):

Available for sale to the public from:

US. Department of Commerce

National Technical Information Service

5285 Port Royal Road

Springfield, VA 22131

Phone: (800) 553-6847

Facsimile: (703) 605-6900

Online ordering: http://www.ntis.gov/ordering.htm

(9) Frinted an renocled patrit 


\title{
ELECTROMIGRATION ISSUES IN HIGH CURRENT HORN*
}

\author{
W. Zhang, S. Bellavia, J. Sandberg, N. Simos, J. Tuozzolo, W-T. Weng \\ Brookhaven National Laboratory, Upton, NY 11973, U.S.A. \\ B. Hseuh, Johns Hopkins University, Baltimore, MD, U.S.A.
}

\section{Abstract}

The secondary particle focusing horn for the AGS neutrino experiment proposal is a high current and high current density device. The peak current of horn is 300 $\mathrm{kA}$. At the smallest area of horn, the current density is near $8 \mathrm{kA} / \mathrm{mm}^{2}$. At very high current density, a few $\mathrm{kA} / \mathrm{mm}^{2}$, the electromigration phenomena will occur. Momentum transfer between electrons and metal atoms at high current density causes electromigration. The reliability and lifetime of focusing horn can be severely reduced by electromigration. In this paper, we discuss issues such as device reliability model, incubation time of electromigration, and lifetime of horn.

\section{INTRODUCTION}

In the proposed Brookhaven AGS Super Neutrino Beam Facility, a magnetic horn will be used to focus the secondary beam. Much attention has been given to physics and mechanical properties of the horn. The electrical and electromechanical issues have not been considered as serious design limitations. All horn problems have been regarded as mechanical failures, material strength limitations, etc. These conclusions are correct in most situations. Our interest is to broaden the investigation and gain understanding of the electromechanical aspect of horn design.

\section{HORN BASIC ISSUES}

The basic physics consideration of horn design is its geometry to capture and focus of secondary particles. The neutrino beam produced will have to travel more than 2500 kilometers to reach the detector at far end. To reduce beam loss, it is desirable to have an ultra thin wall horn to make it as transparent as possible to the secondary beams. The horn geometry is shown in Figure 1.

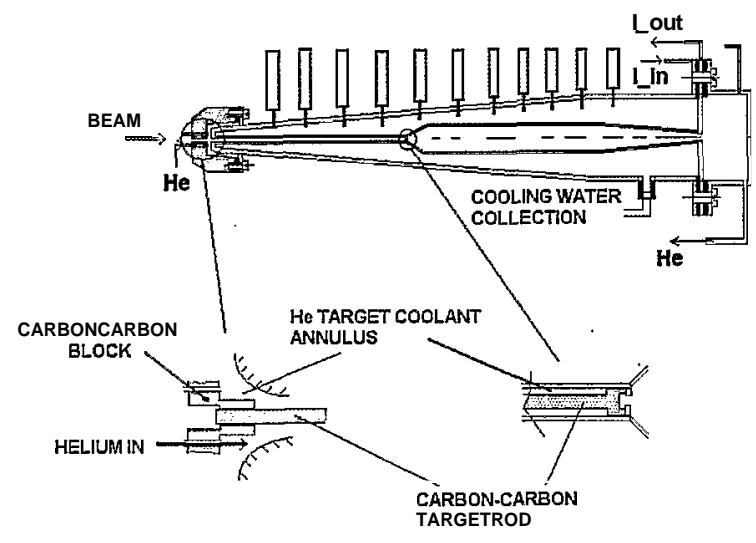

Figure 1. Target and horn configuration.

*Work performed under auspices of U.S.Dept. of Energy.
Mechanical issues related to target and horn design have been well recognized. These include: horn material selection, the horn and target integration, the thermal distribution of horn, the heat removal mechanism, the material swell due to irradiation, material corrosion, material fatigue, etc.

\begin{tabular}{|c|c|c|c|}
\hline Section & Inner Diameter & $\begin{array}{c}\text { Wall } \\
\text { Thickness }\end{array}$ & $\mathrm{L}$ \\
\hline $\mathrm{L} 1$ & $14 \mathrm{~mm}$ & $2.5 \mathrm{~mm}$ & $800 \mathrm{~mm}$ \\
\hline \multirow{2}{*}{$\mathrm{L} 2$} & $\begin{array}{c}14 \mathrm{~mm} \\
\text { minimum }\end{array}$ & \multirow{2}{*}{$2.5 \mathrm{~mm}$} & \multirow{2}{*}{$100 \mathrm{~mm}$} \\
\hline & $\begin{array}{c}120 \mathrm{~mm} \\
\text { maximum }\end{array}$ & & \\
\hline L3 & $120 \mathrm{~mm}$ & $2.5 \mathrm{~mm}$ & $800 \mathrm{~mm}$ \\
\hline \multirow{2}{*}{ L4 } & $\begin{array}{c}120 \mathrm{mmm} \\
\text { maximum }\end{array}$ & \multirow{2}{*}{$1.0 \mathrm{~mm}$} & \multirow{2}{*}{$400 \mathrm{~mm}$} \\
\hline & $\begin{array}{c}12 \mathrm{~mm} \\
\text { minimum }\end{array}$ & & \\
\hline
\end{tabular}

Figure 2 Simplified Horn geometry

At the fundamental frequency, $833 \mathbf{H z}$, of the current pulse, the skin depth is $3.574 \mathrm{~mm}$. The wall thickness of the horn is less than the skin depth. Therefore, we assume the wall is fully saturated by pulse current. At peak current level of $300 \mathrm{kA}$, the current density can reach as 
high as $7.3456 \mathrm{kA} / \mathrm{mm}^{2}$. Figure 3 and 4 show the current conducting area and current density distribution alone horn axial.
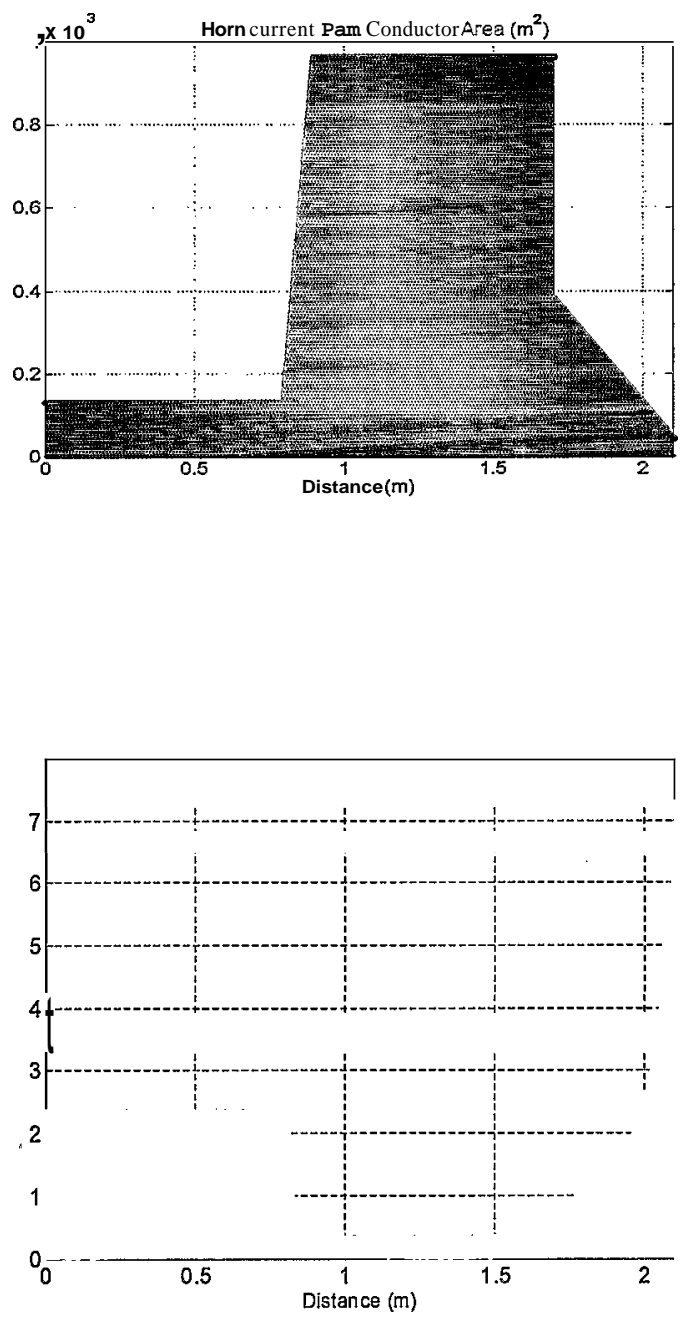

Figure 4. Horn current density

According to various literatures, the discovery of electromigration was more than hundred years ago. The electromigration is due to momentum exchange of electrons and metal atoms. At high current density massive electrons collide with metal atoms, and the effect becomes noticeable. It happens at the place of material voids, impurities, and grain boundaries, etc. Although the electromigration force is proportional to the current density, the electromigrationfailure rate is proportional to higher orders of the current density. One famous result is the Black's equation, which relates the electromigration mean time to failure to the inverse square of the current density:

$$
t_{50}=c \frac{e^{E_{a}}}{J^{2}} .
$$

Where $t_{50}$ is mean time to failure, $c$ is a constant determined from experiment, $\mathrm{E}$, is the activation energy, $\mathrm{k}$ is Boltzmann's constant, $\mathrm{T}$ is temperature, and $\mathrm{J}$ is the current density.
The Black's equation was an empirical result obtained in the sixties. There have been many explanations based on different theories. Most researches are based on experimental results of various materials, different sample geometry, and laboratory setups. A modified version of Black's relation is often used as:

$$
\tau \propto \frac{e^{k T}}{J^{n}} .
$$

Where $\tau$ is device lifetime, and $\mathrm{n}$ is to be determined by experiment.

Aluminum material will be used for horn construction. Experimental results of aluminum material show a wide range of $\mathrm{n}$ from 1.8 to 16 depending on many other variables. However, in the current density range being considered, we might assume $n=2$.

Other factors may also affect the overall reliability of the horn device. In summery, commonly concerned factors include:

3 Current density,

$>$ Ambient temperature,

3 Resistive Joule heating,

3 Water and moisture,

3 Environment,

3 Material swell caused by ionized radiation,

3 Solderingjoints,

3 Material fatigue due to electromagnetic force,

3 Material defects,

$>$ Surface condition,

3 Mechanical stress due to structural factors, etc.

It should be pointed out that the joule heating is an important parameter associated with lifetime of device. Under operating condition of $240 \mathrm{kA}, 1.2 \mathrm{~ms}$ pulse width, $2.5 \mathrm{~Hz}$ repetition rate, the temperature at the tip of horn can reach as high as $470 \mathrm{~K}$. This would mean the area with highest temperature is much more likely to induce electromigration than.the area of relative low temperature of $144 \mathrm{~K}$ : Using Black's equation, this temperature difference of $326 \mathrm{~K}$ will reduce device lifetimeby a factor of $3.67 \times 10^{14}$ in the high temperature region.

The tip of horn is also the area of highest current density. The ratio of its current density to the area with lowest current density is about 23.5 times. When current density square rule is used, it will imply that the device lifetime at horn tip is $\mathbf{5 5 5}$ times less than the large straight section L3.

- The activation energy of the particular aluminum material shall also be determined empirically. For the purpose of discussion, we use the lattice activation energy of $1.4 \mathrm{eV}$, and grain boundary activation energy of $0.6 \mathrm{eV}$ for aluminum alloy [9]. Considering that protons enter target area have particle momentum of $28 \mathrm{GeV}$, it's reasonable to believe any uncontrolled spray of primary beam onto the aluminum wall could cause metal atoms to dislocate. The secondary beam travel through thin wall of horn may also cause material activation. Therefore the beam itself is a contributing factor to electromigration. 
Table 11 lists horn parameters for comparison. It can be seen that the maximum current density of proposed AGS horn is 14.37 to 24.48 times of other devices. The electromigration due to the current density square factor alone would mean a 206 to 500 times degradation in device lifetime.

Table 11Horn current and pulse lifetime to study the horn device reliability and electromigrationis derived from a collection of related research works [2-10].

\section{CONCLUSION}

While this derice. is in proposal stage we would like to
extrapolate the lifetime of other horn devices to estimate the frequency of horn replacement during operation. This shall aid the planning and decision of remote automated

Figure 5. Electromigration and reliability model.

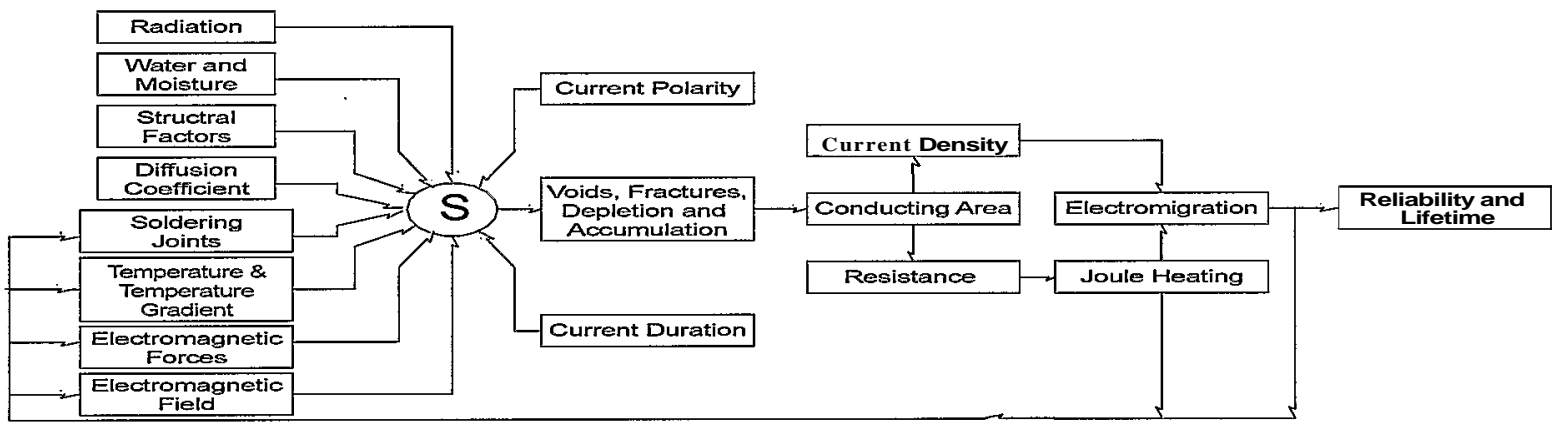

\begin{tabular}{|l|c|r|r|r|c|}
\hline Experiment & $\mathrm{I}_{\text {peak }}$ & $\mathrm{J}_{\text {peak }}$ & $\mathrm{T}_{\text {pulse }}$ & \multicolumn{1}{c|}{$\mathrm{F}$} & $\mathrm{N}_{\text {design }}$ \\
\hline & $\mathrm{kA}$ & $\mathrm{A} / \mathrm{mm}^{2}$ & \multicolumn{1}{c|}{$\mu$} & $\mathrm{Hz}$ & pulses \\
\hline NuMi & 200 & $?$ & 2600 & 0.54 & $\mathrm{I} \times 10^{7}$ \\
\hline MiniBooNe & 170 & 447 & 143 & 5.00 & $1 \times 10^{8}$ \\
\hline K2K & 250 & 511 & & 0.50 & $11 \times 10^{6}$ \\
\hline $\begin{array}{l}\text { Nufact } \\
\text { prototype }\end{array}$ & 300 & 300 & 81 & 50.00 & $2 \times 10^{8}$ \\
\hline CNGS & 150 & 363 & & 0.33 & $42 \times 10^{6}$ \\
\hline $\begin{array}{l}\text { AGS } \\
\text { Superbeam }\end{array}$ & 300 & 7345 & 1200 & 2.50 & $8 \times 10^{6}$ \\
\hline
\end{tabular}

Other studies have shown that the electromigration incubation time factor, bipolar current effect, wet effect, etc. The incubation time is related to void growth, extrusion, edge displacement, and more importantly the flux divergence. The electromigration is a process of mass transfer. It requires discrete time to cause an event to occur. The accumulated pulse time is a production of the number of pulses and pulse duration. Therefore short pulse duration might have advantage of longer horn lifetime.

The force of bipolar current tends to carry the metal atoms back and forth. It has been demonstrated by others that the metal sample tested under the same current density condition last longer with alternative current than direct current. It prompts us to consider if a bipolar wave shape is better rather than half sine.

However, the short pulse width implies higher voltage, and bipolar current is related to bipolar voltage waveform. It will make the high voltage design very difficult, especiallyunder ionized environment.

We are interested on the technology that could extend the horn lifetime and benefit the physics experiment as well. Nano-material bonding has been one of the considerations, and it certainly deserves more research and development. A simple model, as shown in Figure 5, horn handling capability and storage facility.

We are interested in explore new design method, new material composition, and simulation model as well as test approach. More than forty references are used in this work, only a few can be listed due to space limitations.

\section{RE 'EN LES}

[1] M. Diwan, W. Marciano, W. Weng, "AGS Super Neutrino Beam Facility", BNL-7 1228, Internal.

[2] K. Hinode, T. Furusawa, Y. Homma, "Dependence of Electromigration Lifetime on the Square of Current Density", 1993 IEEE/IRPS, pp. 317- 326.

[3] J. Tao, N. W. Cheung, C. Hu, "Modeling Electromigration Lifetime Under Bidirectional Current Stress", IEEE Electron Device Letters, Vol. 16, No. 11, November 1995,pp. 476-478.

[4] C. Basaran, M. Lin, H. Ye, "A Thermodynamic Model for Electrical Current Induced Damage", 2004 Electronic Components and Technology Conference, pp. 1738-1745.

[5] D. Young, A. Christou, "Failure Mechanism Models for Electromigration", IEEE Transactions on Reliability, vol. 43, No. 2, 1994 June, pp. 186-192.

[6] B. T. McCuistian, H. Davis, K. Nielsen, B. Stone, "ATLAS Current Connection Tests Performed on PEGASUS II", Proceedings of 1999 IEEE Pulsed Power Conference, pp. 680-683.

[7] Simeon J. Krumbein, "Metallic Electromigration Phenomena", IEEE Transactions on Components, Hybrids, and Manufacturing Technology, Vol. 11, No. 1, March 1988,pp. 5-13.

[8] M. Sakimoto, T. Itoo, K. Fujii, H. Yamaguchi, K. Eguchi, "Temperature Measurement of A1 Metallization and the Study of Black's Model in High. Current Density", 1995 IEEE, pp. 333-341.

[9] J. R. Lloyd, "Electromigration for Designers: An Introduction for the Non-specialist", TechOnLine. 
[10] M. H. Wood, S. C. Bergman, R. S. Hemmert, "Evidence for an Incubation Time in Electromigration Phenomena", IEEE/IRPS, 1991,pp.70- 76. 